\title{
The Influence of Process Conditions on the Local Shrinkage of the Injection Moulded Natural Fibre Composite with Polypropylene Matrix
}

\author{
Robert Zaboj \\ Department of Engineering Technology \\ Technical University of Liberec \\ Czech Republic
}

\begin{abstract}
Increasing demands on strict geometry and dimensions requirements of plastic parts need systematic research in the injection moulding field with impact on dimension stability.
\end{abstract}

This work is focusing on optimization of the chosen processing parameters and their mutual interaction. The aim is to reach as much as optimal processing conditions in terms of required dimensional stability - rectangular plate - applied on semicrystalline polymer - nature fibres composite with polypropylene homopolymer matrix in modification $(\mathrm{PP}+\mathbf{3 0 \%}$ cellulose $+\mathbf{3 0} \%$ additive Smart)

Although shrinkage is influenced by wide range and combination of the process parameters, there had been chosen three basic parameters on the basis of practical experiences and ambiguity melt temperature, holding pressure and injection rate.

Keywords-injection moulding; shrinkage; cavity pressure; polypropylene

\section{INTRODUCTION}

Injection moulding is one of the most important technology of thermoplastic polymer processing. More than $30 \%$ of plastic parts worldwide are produced by that technology. Thermoplastic products can be manufactured as large-lot production of semi products as well as final products of various shapes and sizes. Some technical application required tight dimensioned tolerances first of all in automotive industry. Plastic parts are assembled into more complex technological sets therefore must be produced in tight dimensional specifications. Product's final properties are outcome from various factors as resin composition, mould and product design and processing conditions[1].

Shrinkage is one of the most significant attribute determining final product's dimensions. Semicrystalline polymers show higher shrinkage in comparison with amorphous. Contrary to amorphous semicrystalline show sharp transition between rubber state and amorphous melt caused by melting the crystals. Crystallization comprises local arrangement of short lengths (segments) of adjacent chains. These crystal structures grow during the cooling of melt polymer inside the mould cavity. Crystallization is a volumereduction process thus semicrystalline polymers show higher values of shrinkage. Another factor influencing shrinkage is molecular weight. High molecular-weight polymers exhibit higher viscosity during filling stage. That caused higher pressure drop inside mould cavity and high injection pressure is required to compensate shrinkage[1,2].

Process variables as melt temperature, injection rate, holding pressure and time, mould temperature are another crucial factors influencing shrinkage. Effect of these variables is not only dependent on reciprocal interaction among each other but also on material, mould and product design [2].

Mould temperature influencing cooling rate and then resulting degree of crystallinity. The higher cooling rate, the less time for arrangement of molecular chains and the smaller shrinkage [3].

Effect of melt temperature is ambiguous. From shrinkage aspect could be observed two opposed effects -1 . The higher melt temperature, the higher thermal contraction and the higher shrinkage -2 . The higher melt temperature, the lower viscosity and the better pressure response which leads to reduction of shrinkage [2].

Injection rate has similar ambiguous effect as melt temperature. Higher injection rate caused secondary temperature increase of melt by high shear stress and then better pressure response in mould cavity. Friction heat result from gate restrains and between flowing material in the core of pressed piece and solidified surface near the mould wall. However orientation and reorientation effects react against it as well as distribution of internal strain and inconstant viscosity. Low injection rate needs higher melt temperature to sufficient mould filling which can leads to better pressure response and shrinkage reduction[2].

Holding pressure and time has significant influence on shrinkage compensation. The higher cavity pressure during gate solidification the higher pressure response in injected part and then smaller shrinkage. Pressure value is changing with increasing distance from gate. Maximal value is measured near gate and decreasing along flow path. The pressure difference between the gate and the end of flow has crucial effect especially for long flow path or thin-wall parts[4, 5]. 


\section{MATERIAL AND EXPERIMENTAL}

\section{A. Material}

Composite with natural fibre additives and semicrystalline thermoplastic matrix was used in this work. Matrix Homopolymer Polypropylene Thermofil E020M - natural cellulose fibres in contents $30 \%$ and additive Smart from Dow company.

\section{B. Machine}

The test was carried out on injection machine ARBURG ALLROUNDER 570 C 2000-675

\section{Mould}

The special mould for observation rheological, temperature and pressure relations was used. That measurement is ensured by three pressure transducers located inside cavity, which scanning pressure evolution during injection. Samples produced such mould ensuring comparable process conditions during injection and then results of measurement providing higher reliability. Rectangular cavity with nominal thickness $2 \mathrm{~mm}$ was used. The cavity was filled with fan gate located in upper part of test segment in whole its length to achieve balanced filling of cavity.

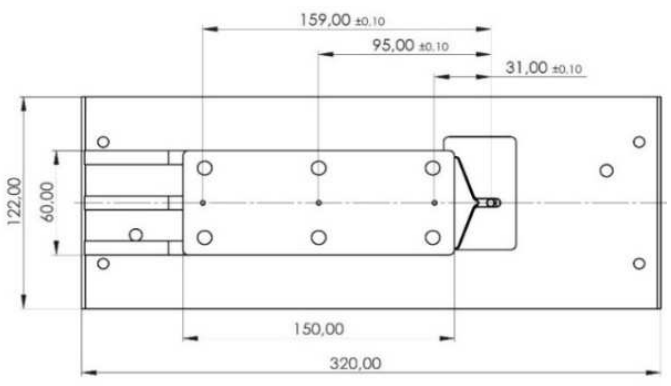

\section{FIGURE I. MOULD CAVITY WITH NOMINAL DIMENSIONS} flow.

The traducers were located near the gate and at the end of

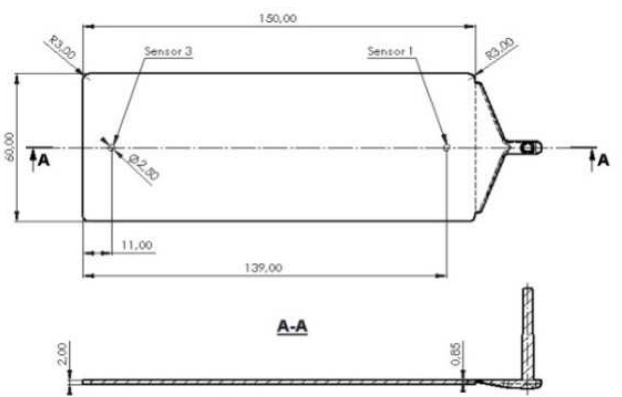

FIGURE II. DIMENSIONS AND SENSOR LOCATION OF TEST RECTANGULAR PLATE

\section{Process Conditions and Measurement}

There were chosen three process parameters - melt temperature, holding pressure and injection rate. Each parameter was chosen at three levels which are commonly used in industry. The mould cavity had been set on nominal thickness $2 \mathrm{~mm}$, which is medium-value in range of thickness used in industry.

For optimal combination of the process parameters was used standard orthogonal array L9.

\begin{tabular}{|l|l|l|l|}
\hline $\begin{array}{c}\text { Factor } \\
\text { Experiment }\end{array}$ & $\begin{array}{c}\text { Holding } \\
\text { Pressure }\end{array}$ & $\begin{array}{c}\text { Melt } \\
\text { Temperature }\end{array}$ & $\begin{array}{c}\text { Injection } \\
\text { Rate }\end{array}$ \\
\hline 1 & 1 & 1 & 1 \\
\hline 2 & 1 & 2 & 2 \\
\hline 3 & 1 & 3 & 3 \\
\hline 4 & 2 & 1 & 2 \\
\hline 5 & 2 & 2 & 3 \\
\hline 6 & 2 & 3 & 1 \\
\hline 7 & 3 & 1 & 3 \\
\hline 8 & 3 & 2 & 1 \\
\hline 9 & 3 & 3 & 2 \\
\hline
\end{tabular}

TABLE I. ORTHOGONAL ARRAY OF THE EXPERIMENT

The other process condition which are not object of investigation were held as constant. The holding time was determined during initial phase of the experiment for maximal weight of testing sample for given combination of parameters. Cooling medium temperature was set up according to technical data sheet of the resin. Five samples were taken after stabilizing of the process for each batch. The samples were kept in a room with constant temperature $\pm 23^{\circ} \mathrm{C}$ and measured after 48 hour period.

The thickness dimensions were measured in position B1 (sensor position near gate), position B2 (middle of flow) and position B3 (sensor position end of flow). Shrinkage was calculated as $\frac{\mathrm{L} 0-\mathrm{L}}{\mathrm{L} 0}$.

Where Lo is the mould insert dimension and $\mathrm{L}$ is the dimension of the sample.

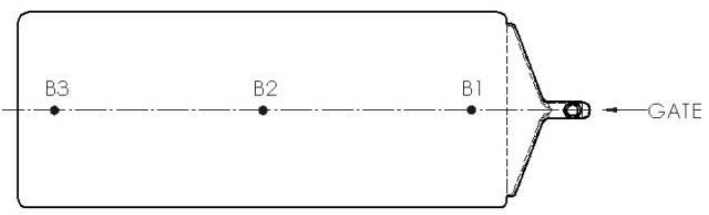

FIGURE III. MEASURED POSITIONS

\begin{tabular}{|l|l|}
\hline $\begin{array}{l}\text { Cooling Medium temperature } \\
\left({ }^{\circ} \mathrm{C}\right)\end{array}$ & $30^{\circ} \mathrm{C}$ \\
\hline Melt Temperature $\left({ }^{\circ} \mathrm{C}\right)$ & $190 / 200 / 210$ \\
\hline Clamping Force $(\mathrm{kN})$ & 2000 \\
\hline Feed Path $(\mathrm{ccm})$ & 55 \\
\hline Injection Rate $\left(\mathrm{ccm} . \mathrm{s}^{-1}\right)$ & $20 / 100 / 200$ \\
\hline Holding Pressure Switch Point & $100 \%$ sample volume \\
\hline Holding Pressure (HP) & $\begin{array}{l}80 / \text { 90 } / 100 \% \\
\text { injection pressure at } \\
\text { HP switch point }\end{array}$ \\
\hline Holding time (s) & 13 \\
\hline
\end{tabular}

TABLE II. EXPERIMENTAL PROCESS CONDITIONS 


\begin{tabular}{|l|l|}
\hline \multicolumn{1}{|c|}{$\begin{array}{c}\text { Oder of Set Up Parameters: } \\
\text { HoldingPressure- } \\
\text { HP(bar)MeltTemperature }\left({ }^{\circ} \mathbf{C}\right) / \text { Injection } \\
\text { Rate }\left(\mathbf{c c m}^{-1} \mathbf{s}^{-1}\right)\end{array}$} & $\begin{array}{c}\text { Real values } \\
\text { of the } \\
\text { holding } \\
\text { pressure }(\text { bar })\end{array}$ \\
\hline $80 / 190 / 20 \quad$ & 474 \\
\hline $80 / 200 / 100$ & 602 \\
\hline $80 / 210 / 200$ & 790 \\
\hline $90 / 190 / 100$ & 726 \\
\hline $90 / 200 / 200$ & 910 \\
\hline $90 / 210 / 20$ & 454 \\
\hline $100 / 190 / 200$ & 1026 \\
\hline $11 / 200 / 20$ & 585 \\
\hline $100 / 210 / 100$ & 705 \\
\hline
\end{tabular}

TABLE III. ORDER OF SET UPPARAMETERS

\section{RESUlTS}

\section{A. Cavity Pressure Evolution for the Different Holding Values}

Effect of processing parameters on experimental pressure evolution inside the mould cavity is captured on following graphs. Pressure curves are time function for particular combination of the processing parameters (melt temperature, injection rate, holding pressure).Pressure curves provide more detail information concerning influence of pressure inside the mould.

From the graphs can be seen, that pressure traces are linked with different sensor position. Cavity pressure rises from zero level when melting material reach sensor 1 and sensor 3 .

At injection rate below $100 \mathrm{ccm} / \mathrm{s}-1$ combined with melt temperature up to $200^{\circ} \mathrm{C}$ cavity pressure reach zero at time interval between $8-10 \mathrm{~s}$. At this time the gate is already freeze- off and shrinkage of material starts. Low injection rate together with low melt temperature (80/190/20) induce rapid pressure drop down to zero from maximal value 287 bar measured near the gate at the time $8,1 \mathrm{~s}$. At that moment the gate is freeze-off and holding pressure effect is restrained. Injection rate $100 \mathrm{ccm} / \mathrm{s}-1$ and melt temperature $200^{\circ} \mathrm{C}$ $(80 / 200 / 100)$ enhance pressure response in position near the gate only (see pressure curve for position A). Pressure curves $(80 / 210 / 200)$ show that high melt temperature and injection rate make non-uniform pressure distribution in cavity. At the end of flow the pressure reach zero at $11,3 \mathrm{~s}$. while near the gate the pressure effect is extended to $22,2 \mathrm{~s}$.

(90/190/100) captured pressure reach zero in position 1 (near the gate) at $10,8 \mathrm{~s}$.

(90/210/20) After reaching pressure peak there is obvious gradual drop down to gate free-off in position 1 (near the gate). That is probably caused by high melt temperature $210^{\circ} \mathrm{C}$ extending solidification process and pressure response.

(90/200/200) There is obvious considerable difference of pressure drop down to zero between position 1 and 3 . In position 3 pressure reaches zero at $14,8 \mathrm{~s}$, while in position 1 can be observed residual non-zero value due to polymer over packing at the moment gate freeze-off and thermal contraction is not able exceed pressure effect.
Maximal holding pressure value $(100 \%)$ prolongs pressure effect to $16,2 \mathrm{~s}$ with injection rate $100 \mathrm{ccm} / \mathrm{s}-1$, resp. $12,1 \mathrm{~s}$ with $20 \mathrm{ccm} / \mathrm{s}-1$.

$(100 / 210 / 100)$ It is noticeable gradual pressure drop near the gate (position 1) and relatively wider zero-value time interval between position 1 and 3 .

(100/190/200) There can be observed residual pressure for both positions after the end of the cycle and mould opening.

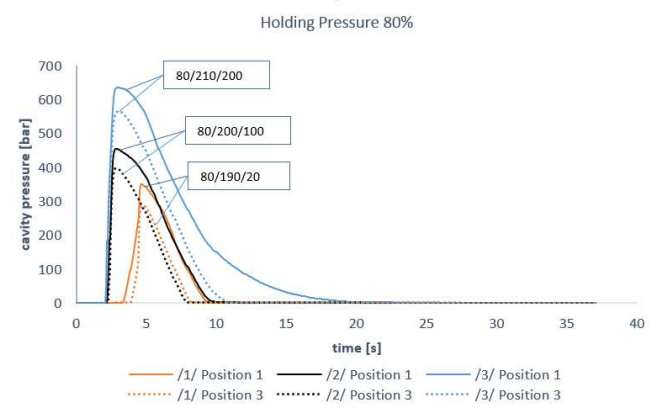

FIGURE IV. PRESSURE EVOLUTION - HP $80 \%$



FIGURE V. PRESSURE EVOLUTION - HP 90\%

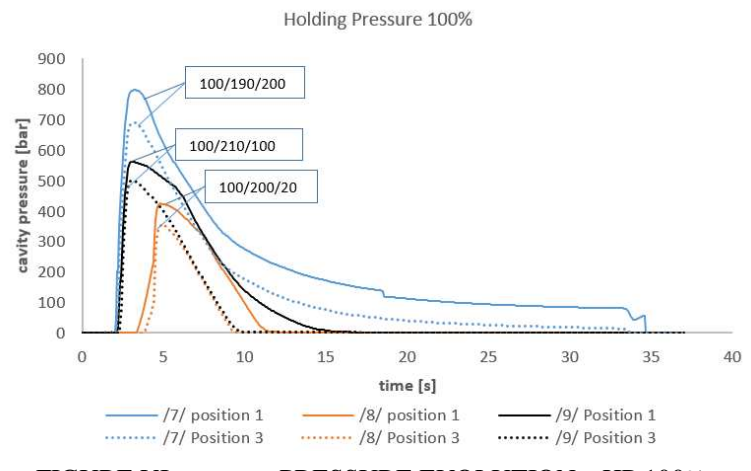

FIGURE VI. PRESSURE EVOLUTION - HP $100 \%$

\section{B. Shrinkage for the Different Holding Values}

Shrinkage was measured in different moulding parameters (identical combination as in pressure monitoring in previous paragraph). In this section the effect of combination processing conditions on shrinkage is analysed. Some combinations show negative values of shrinkage which is undesirable in practice.

(80/190/20) Maximal value of local shrinkage in extent $1,40-5,49 \%$ linearly rising from the gate to the end of the 
flow. Low value of packing and low injection rate make earlier gate freeze-off and restrain holding phase effect.

(80/200/100) Samples showing moderately negative shrinkage (dimension enlargement) near the gate (position $\mathrm{A}$ ) then rapidly growing in the middle of flow (position $\mathrm{B}$ ) up to $4,6 \%$ at the end of flow (position C). This could be explained since pressure holding effect decreasing with growth distance from the gate which causes non-linear shrinkage.

(80/210/200) There is significant difference of local shrinkage between the positions near the gate and at the end of flow. This is due to high injection rate and high melt temperature make packing more efficient. Local shrinkage is negative $-2,79 \%$ near the gate and 2,89 at the end of flow.

(90/190/100) Maximal value of shrinkage reach $4,18 \%$ at the end of flow and almost linearly decline down to negative value $-0,89 \%$. Injection rate $100 \mathrm{ccm} / \mathrm{s}-1$ and holding $726 \mathrm{bar}$ cause local over packing near the gate. Across the flow pressure transfer is reduced probably by contribution of lower melt temperature.

(90/210/20) Low injection rate makes faster solidification process at the end of flow which reduces pressure transfer despite the highest melt temperature. Maximal shrinkage 5,19 was measured at the end of flow and descending down to $0,67 \%$ near the gate where is holding more effective as a result of high melt temperature.

(90/200/200) These parameters show most shrinkage difference between the position A near gate 3,69\%) and position $\mathrm{C}$ at the end of flow (2,33\%). In position $\mathrm{C}$ material starts rapidly cool and after pressure reach zero start shrinkage. To the contrary in position A the melt is warmer due to effect of friction heat arise from high injection rate. Combination with holding 909 bar causes local over packing.

Maximal holding values decreasing shrinkage in all combination of parameters.

$(100 / 200 / 20)$ Shrinkage $(4,8 \%)$ occurs at the end of flow. Near the gate maximal holding forces pressure effect into melt which resulting in low negative shrinkage $(-0,08 \%)$.

(100/210/100) The high values of holding and melt temperature prolong pressure effect near the gate where negative shrinkage $(-1,82 \%)$ was measured.

(100/190/200) High injection rate and holding pressure probably deforming individual parts of mould and lead to significant negative shrinkage at the end and in the middle of flow.

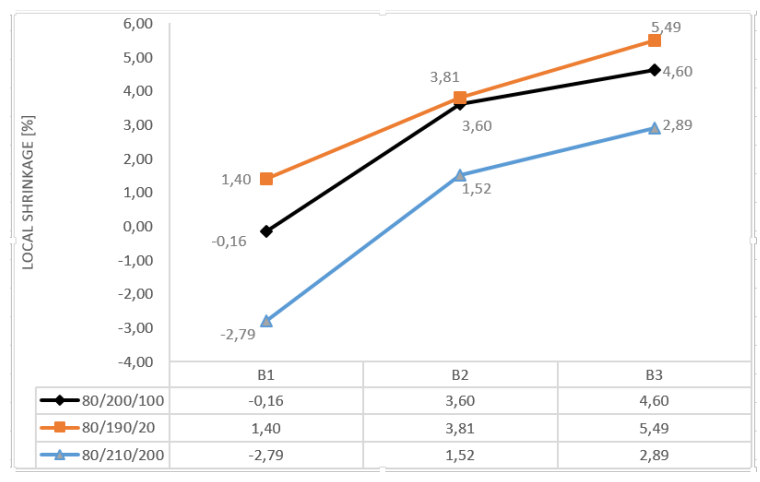

FIGURE VII. SHRINKAGE HP $80 \%$

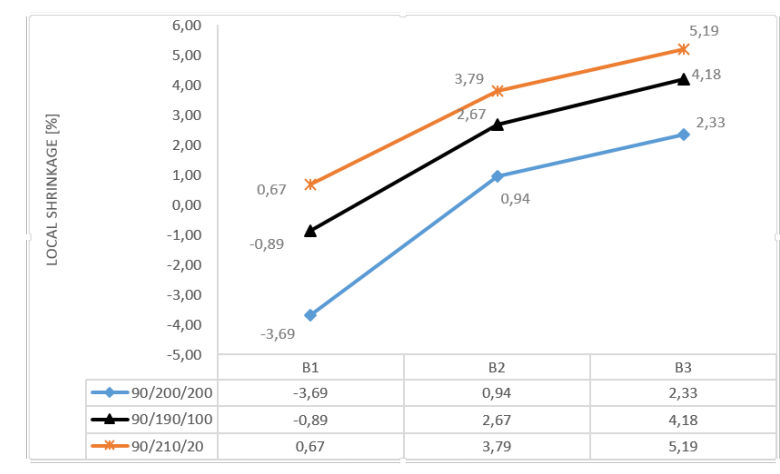

FIGURE VIII. SHRINKAGE HP $90 \%$

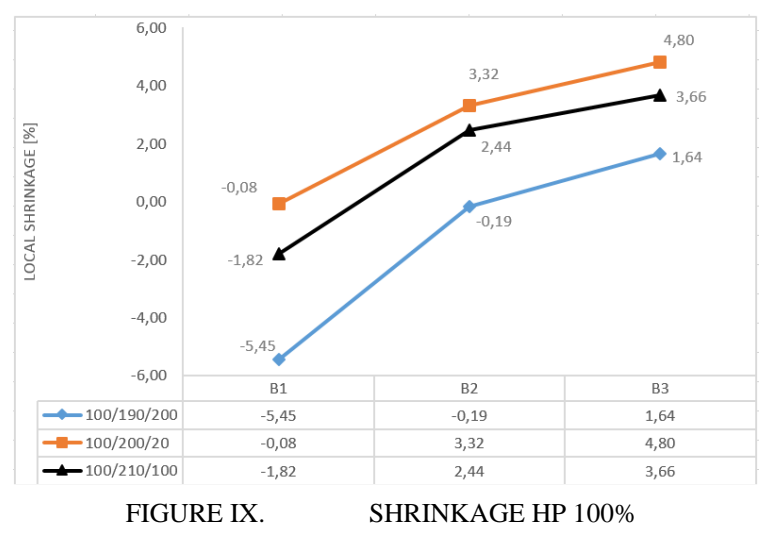

IV. CONCLUSION

On the basis of the experiment it is possible to state that shrinkage grows with the length in the direction of flow. It is evident that local shrinkage is decreasing to negative values of shrinkage with increasing pressure inside the mould. Increase of holding pressure and melt temperature caused local shrinkage decrease. Higher melt temperature decreases material viscosity and extending solidification process as well as time of the pressure effect in cavity. That leads to higher values of pressure integral during holding phase. Lower values of holding cause rapid pressure drop inside cavity and reach zero value before mould opening.

All combinations with injection rate 200 results in great differences of rest pressure in cavity between areas of the gate and end of flow. Mould over packing and considerable negative shrinkage by the gate is the result of all the above 
cases. The polymer is over packed in the moment of gate freeze-off and thermal contraction initialized by cooling is not able to overcome rest pressure effect. Friction heat evolving during melt passing through the gate by high injection rate also contributes to that fact. In the area of the gate friction heat decreasing viscosity of material arises, which extends holding phase and therefore improves pressure response. Negative shrinkage (dimension enlargement) is probably caused by elastic expansion of pressure polymer in the gate and by mould deformation. The highest values of local shrinkage were measured for all set up combinations with the lowest injection rate $20 \mathrm{ccm} / \mathrm{s}-1$.

\section{REFERENCES}

[1] Greener, J.; Wimberger-Friedl, R. - Precision Injection Molding: Process, Material, and Applications - Carl Hanser Verlag, Munich 2006

[2] Fischer, Jerry M.; Handbook of molded part shrinkage and warpage Plastic Design Library - ISNB 1-884207-72-3

[3] Gipson, P.M. Grelle, P.F., and Salamon, B.A. - The Effect of Process Conditions, Nominal Wall Thickness, and Flow Length On The Shrinkage Characteristics of Injection Molded Polypropylene - The Dow Chemical Company, Midland, Michigan

[4] Jansen, K.M.B; van Dijk D.J. and Burgers, E.V. - Experimental Validation of Shrinkage Predictions for Injection Molded Parts - Twente University, Dept. Mechanical Engineering, Enschede, The Netherlands

[5] Kuek, Soon-Chun and Angstadt, D.C. - In- Situ Cavity Pressure Monitoring In Micro-Injection Moulding - Department of Mechanical Engineering, Clemson University, Clemson, SC 